\title{
Risk factors for deterioration of renal function after percutaneous nephrolithotomy in solitary kidney patients with staghorn calculi
}

\author{
Jiahao Wang", Yunjin Bai", Shan Yin, Jianwei Cui, Yin Tang, Zhenghao Wang, Bo Chen, Hong Li, \\ Wuran Wei, Jia Wang \\ Department of Urology, Institute of Urology, West China Hospital, Sichuan University, Chengdu, China \\ Contributions: (I) Conception and design: J Wang; (II) Administrative support: None; (III) Provision of study materials or patients: J Wang, Y Bai; (IV) \\ Collection and assembly of data: J Wang, J Cui; (V) Data analysis and interpretation: J Wang, Y Bai; (VI) Manuscript writing: All authors; (VII) Final \\ approval of manuscript: All authors. \\ "These authors contributed equally to this work. \\ Correspondence to: Jia Wang. Department of Urology, Institute of Urology, West China Hospital, Sichuan University, Chengdu, China. \\ Email: wangjiawch@163.com.
}

Background: Nowadays, it has not been reported in detail which factors have adverse effects on renal function after PNCL surgery in solitary kidney patients with staghorn calculi. To evaluate the risk factors that deteriorate the renal function in solitary kidney patients with staghorn calculi after percutaneous nephrolithotomy (PCNL).

Methods: A retrospective study was performed on solitary kidney patients with staghorn calculi between March 2014 and Jun 2019. Renal function-related indexes were collected pre-operatively, and at 1 and 6 months post-operatively. These pre- and post-operative indexes were compared to study the risk factors on renal function.

Results: Totally 71 solitary kidney patients with staghorn calculi were included with a male-to-female ratio of 53:18 and mean age of $54.3 \pm 10.2$ years old. The mean pre-operative estimated glomerular filtration rate $(\mathrm{eGFR})$ and serum creatinine were $55.1 \pm 18.9 \mathrm{~mL} / \mathrm{min} / 1.73 \mathrm{~m}^{2}$ and $130.9 \pm 41.0 \mu \mathrm{mol} / \mathrm{L}$, respectively. The mean diameter of largest stones was $5.0 \pm 1.7 \mathrm{~cm}$, and the stone-free rate was $71.8 \%$. Univariate analysis showed urinary tract infection (UTI) was a significant risk factor for deteriorating renal function after PCNL. At 1 to 6 months after surgery, renal function deteriorated by both UTI and combined diseases. The surgical side at the right kidney, mild hydronephrosis, and surgical time without 20 min or beyond $40 \mathrm{~min}$ can deteriorate renal function. Multivariate analysis demonstrated UTI was the main factor leading to deteriorated renal function with increasing serum creatinine and declining eGFR.

Conclusions: Preoperative UTI can deteriorate the renal function at 1 month after PCNL, and diabetes can deteriorate the postoperative renal function at 6 months after PCNL. Controlled perioperative UTI, diabetes and factors play a crucial role in protecting renal function after PCNL for solitary kidney patients with staghorn calculi.

Keywords: Percutaneous nephrolithotomy (PCNL); renal function; risk factors; solitary kidney; staghorn calculi

Submitted May 07, 2020. Accepted for publication Sep 04, 2020.

doi: 10.21037/tau-20-916

View this article at: http://dx.doi.org/10.21037/tau-20-916 


\section{Introduction}

Urolithiasis is a common disease of the urinary system. The incidence of renal stones is around $10-20 \%$ (1) and is still rising through the changes of living habits and the increasing prevalence of related medical conditions, such as metabolic syndrome, obesity and diabetes mellitus. Renal stones may bring about a loss of renal function and increase the risk of papillary renal cell carcinoma (2). Therefore, it is necessary to actively manage stones to avoid adverse consequences.

Staghorn calculi remain a challenge for urologists, especially in some special situations, such as solitary kidney, ectopic kidney, and horseshoe kidney. Percutaneous nephrolithotomy (PCNL), a commonly-performed minimally invasive procedure, has been recommended as the first-line treatment for staghorn calculi because of the higher stone-free rate (SFR) achieved with minimal morbidity (3). Normal renal function is more important for solitary kidney patients. As an operation requiring kidney puncturing, however, PCNL unavoidably can damage renal function to certain degree. A recent study demonstrates that PCNL is safe and effective for solitary kidney patients and can improve renal function at the post-operative sixth month (4). However, it has not been reported in detail which factors have adverse effects on PNCL in solitary kidney patients with staghorn calculi.

In general, some combined diseases, such as diabetes, hypertension and repeated urinary tract infections (UTI), may affect renal function in the long run by changing the vascular structure and affecting local blood supply, causing local inflammation to change local metabolism (5-7). For patients with renal calculi, renal function can be improved after surgery in most cases, but whether there are some factors deteriorating the renal function of solitary kidney patients with staghorn calculi after PCNL is still unclear.

Taking into account this knowledge, we aim to evaluate the risk factors that deteriorate the renal function in solitary kidney patients with staghorn calculi after PCNL. These risk factors can be used to guide reasonable control in clinical works and to protect renal function after PCNL in solitary kidney patients with staghorn calculi. We present this article in accordance with the STROBE reporting checklist (available at http://dx.doi.org/10.21037/tau-20-916).

\section{Methods}

\section{Patients}

The present study was conducted with the approval and supervised by the ethics committee of West China Hospital [registration number 2018(182)]. The study has conformed to the provisions of the Declaration of Helsinki (as revised in 2013). Because of the retrospective nature of the research, the requirement for informed consent was waived.

Clinical data over a 5.5-year-period from March 2014 to June 2019 were recorded from consecutive solitary kidney patients with renal staghorn calculi who underwent PCNL. The preoperative medical records of the patients were closely followed and their renal functions were followed up in the first and sixth months after PCNL. Abdominal X-ray, abdominal computed tomography (CT), emission computed tomography and diuresis renography were used to evaluate the solitary kidney patients with renal staghorn calculi for inclusion into this study.

The following data were collected and analyzed: demographics, preoperative (pre-op) renal function, renal functions at 1 and 6 months after PCNL, routine urine test, urine culture, surgical side (right or left), operation time (min), degree of hydronephrosis, diameter of largest stone $(\mathrm{cm})$, and combined diseases (mainly diabetes and hypertension).

\section{Definition of renal function and UTI}

\section{Renal function}

Serum creatinine (Scr) and estimated glomerular filtration rate (eGFR) were used to evaluate pre-operative and post-operative renal functions. Data of Scr and eGFR were obtained from the clinical reference range given by the clinical laboratory of the tested Hospital. The Scr reference interval was: males: $68-108 \mu \mathrm{mol} / \mathrm{L}$ ( $>18$ years old); females: 48-79 $\mu \mathrm{mol} / \mathrm{L}$ (18-60 years old) and 49$88 \mu \mathrm{mol} / \mathrm{L}$ ( $>60$ years old). The eGFR reference interval was $56-122 \mathrm{~mL} / \mathrm{min} / 1.73 \mathrm{~m}^{2}$. Changes in Scr or eGFR greater than the normal range were considered to be significant. Since Scr is easily affected by factors such as eating, we evaluated the changes of renal function by integrating the changing trends of the two indexes, so as to avoid the occurrence of evaluation errors. These two indicators were used only to assess whether the patient's renal function had deteriorated.

\section{UTI}

According to the reference standard of the medical laboratory of the tested Hospital, UTI is considered as white blood cell count in the urine $>5 / \mathrm{HP}$, number of bacteria $>230 / \mu \mathrm{L}$, positive nitrite, positive pus cells, and 
positive urine culture or obvious clinical symptoms of infection.

\section{Surgical procedures}

The skin to calyx approach was obtained by gradual dilation of metal dilators to Fr 18. Lithotripsy of staghorn calculi was performed by one surgeon using a Swiss lithoclast (EMS Electro Medical System, Nyon, Switzerland). Considering the comprehensiveness of staghorn calculi, we inserted one Fr 14 nephrostomy tube and one Fr 4.7-6 double J stent into each patient and extracted them on 5 days to 2 weeks postoperatively (8).

\section{Statistical analysis}

Data was recorded on an excel spreadsheet (Microsoft) and all statistical analyses were undertaken using IBM SPSS Statistics 22.0. Means were compared using $t$-test and oneway analysis of variance (ANOVA). The univariate and multivariate binary logistics regression analysis were used to analyze the risk factors. A two-sided $\mathrm{P}<0.05$ was considered to be statistically significant.

\section{Results}

Over the period of 5.5 years, 71 solitary kidney patients suffering staghorn calculi were included with a male:female ratio of 53:18 and a mean age of 54.3 years (rang, 24-71; SD: $\pm 10.2)$. The mean pre-op Scr and eGFR were $130.9 \mu \mathrm{mol} / \mathrm{L}$ (rang, 61-243; SD: \pm 41.0 ) and $55.1 \mathrm{~mL} / \mathrm{min} / 1.73 \mathrm{~m}^{2}$ (rang, 20.3-97.5; SD: \pm 18.9 ) respectively. The mean diameter of largest stone was $5.0 \mathrm{~cm}$ (rang, 3-9.3; SD: \pm 1.7 ), and SFR was $71.8 \%$ (Table S1). And we analyzed all the patients by grouping them into different risk factors.

The mean eGFR and Scr levels characteristic of all subjects under different risk factors are shown in Table 1. The differences of renal function among pre-op, one-month after PCNL and 6 months after PCNL were analyzed by paired-samples $t$-test. At 1 month after PCNL, the Scr level and eGFR of UTI(+) patients accompanied by urine culture $(+)$ showed the most significant changes $(\mathrm{P}<0.01$, $95 \%$ CI: -25 to -10.2$)$, and both indexes reflected a gradual deterioration of renal function. Using ANOVA, the mean value of the difference between per-op and 1 month after PCNL in both Scr $(\mathrm{P}=0.004)$ (Figure 1A) and eGFR $(\mathrm{P}=0.018)$ (Figure $1 B)$ were statistical significance, showing the trend of deterioration of renal function. At 6 months after PCNL, eGFR significantly decreased in patients with diabetes ( $\mathrm{P}=0.037,95 \%$ CI: $0.3-4.3$ ). Meanwhile, eGFR also significantly declined at the operation time within $20 \mathrm{~min}$ ( $\mathrm{P}=0.029,95 \% \mathrm{CI}: 0.1-1.4)$ and beyond $40 \mathrm{~min}(\mathrm{P}=0.029$, 95\% CI: 0.1-1.4). Interestingly, in patients undergoing PCNL on the right side after 6 months, renal function reflected by Scr level or eGFR significantly deteriorated. Although the effect of hydrops on Scr level or eGFR was not significant, there was still a tendency to deteriorate renal function.

To incorporate as many suspected confounders as possible into the model for adjustment, we set $\mathrm{P}<0.2$. Scr level and eGFR were used to jointly evaluate the renal function of patients, and renal function deterioration and no deterioration was used as outcome indicators in univariate and multivariate analyses to compare the ratios of risk factors under these two indicators. The univariate and multivariate analysis of all the risk factors in renal function are presented in Tables 2,3. Factors involved in the univariate analysis included UTI(+), UTI(-), UTI(+) + urine culture(+), UTI(+) + urine culture(-), diabetes, hypertension, operation time, operation side, and degree of hydronephrosis. In the first month after PCNL, $\mathrm{UTI}(+)$ was the major risk factor for postoperative renal function deterioration in both Scr level (OR 3.365, 95\% CI: $1.268-8.929, \mathrm{P}=0.015)$ and eGFR (OR 3.846, 95\% CI: 1.434-10.314, $\mathrm{P}=0.007)$, and $\mathrm{UTI}(+)+$ urine culture $(+)$ can deteriorate renal function in both Scr level (OR 7.636, 95\% CI: 2.251-25.909, $\mathrm{P}=0.001$ ) and eGFR (OR 7.200, 95\% CI: 2.111-24.559, $\mathrm{P}=0.002)$. Interestingly, in the 6 months after PCNL, combined diseases showed a trend of renal function deterioration in both Scr level (OR 3.652, 95\% CI: $1.153-11.565, \mathrm{P}=0.028)$ and eGFR (OR 3.375, 95\% CI: 1.067-10.678, P=0.038), and UTI(+) + urine culture (+) still seemed to have some influence. In the multivariate analysis, compared the renal function between pre-op and 1 month after PCNL, UTI(+) + urine culture(+) showed an increase of Scr level (OR 10.79, 95\% CI: 2.5-45.6) and decline of eGFR (OR 12.3, 95\% CI: 2.9-51.7), both significantly $(\mathrm{P}=0.001)$, coming a trend of renal function deterioration, and showed the same effects on renal function deterioration even in 6 months after PCNL. Other factors appeared to play a role in affecting renal function, but insignificantly.

\section{Discussion}

It is necessary for solitary kidney patients to protect their renal function. We found most solitary kidney patients had 


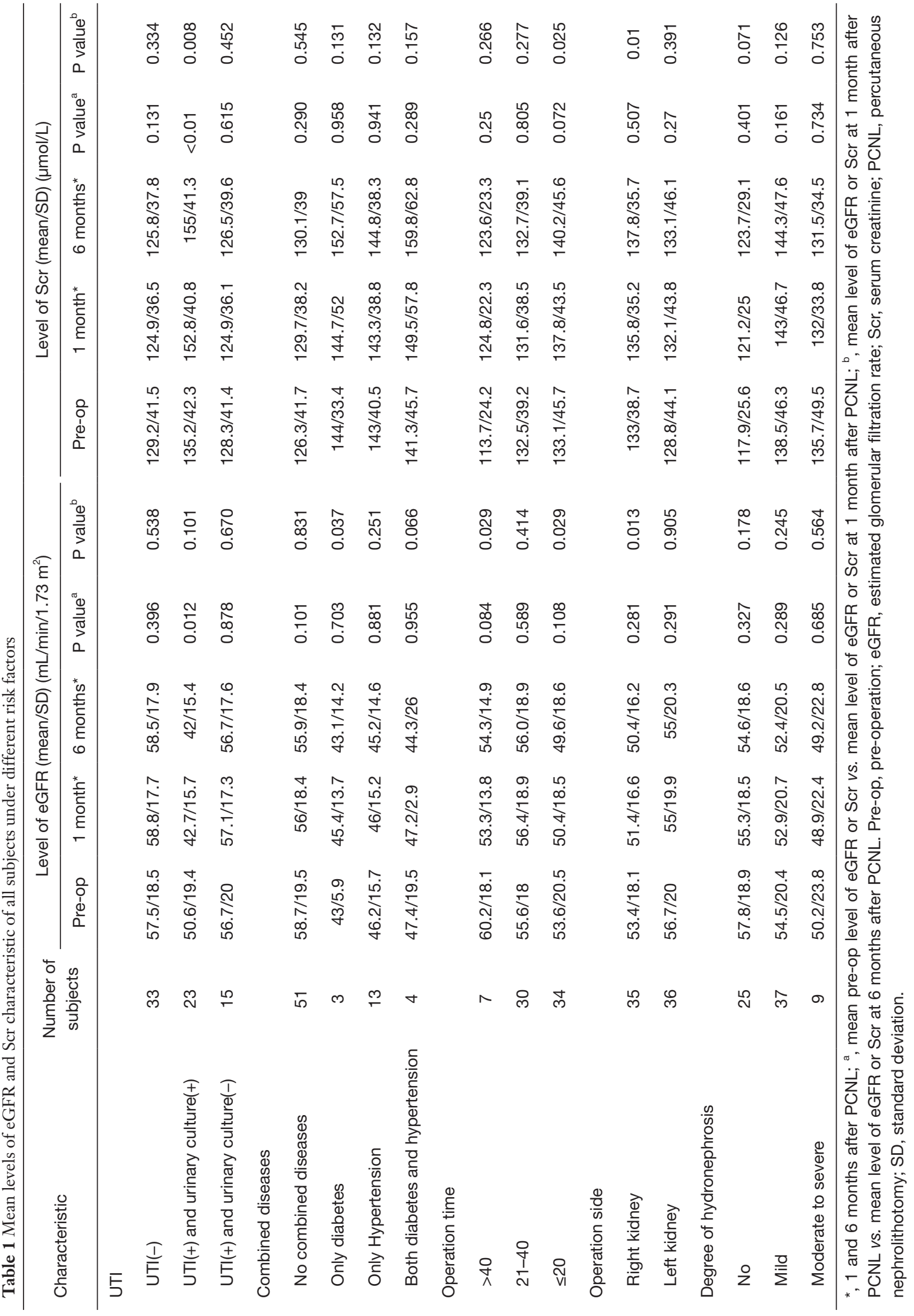



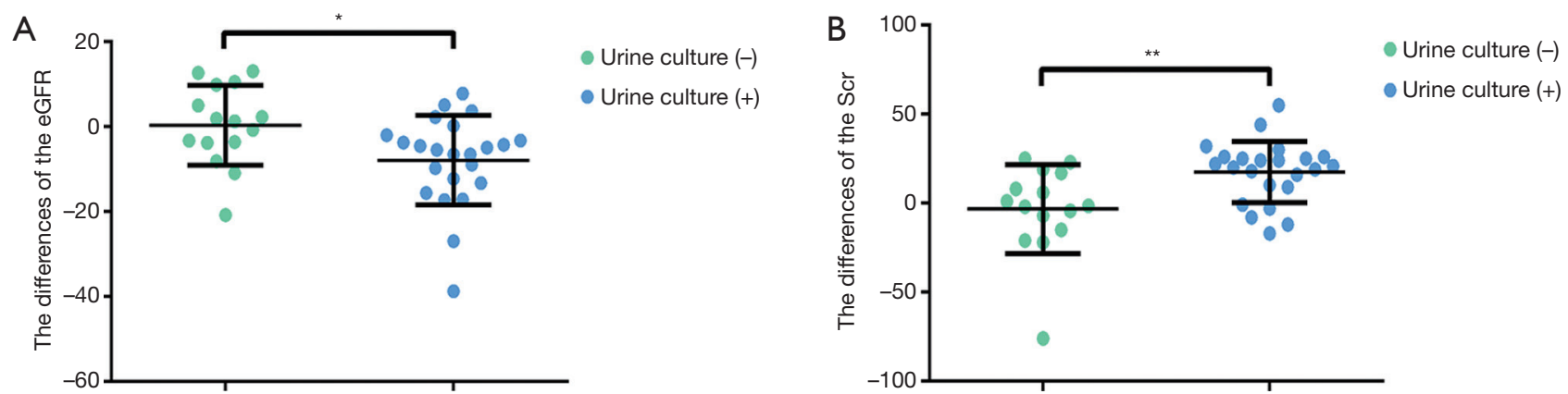

Figure 1 Comparing the differences of eGFR and Scr level in urine culture group. (A,B) Comparing the differences of eGFR and Scr level between pre-op and 1 month after PCNL in urine culture (-) and urine culture $(+)$. ${ }^{*}, \mathrm{P}<0.05 ;{ }^{* *}, \mathrm{P}<0.01$.

no significant change in renal function after PCNL, and even some patients had significant improvement in renal function. However, infection and combined diseases may impede postoperative renal function improvement.

Urinary calculi are often accompanied by UTI, which can lead to local symptoms such as frequent and urgent urination, and the combination with severe UTI even can cause urogenic sepsis. It is well-known that local inflammation and infection are not conducive to wound repair $(9,10)$. We found UTI deteriorated the renal function at 1 month after PCNL. As an invasive operation, PCNL will inevitably cause local bleeding, and upon operative area infection, bacteria can enter the bloodstream from these damaged wounds and cause local inflammation. Local accumulation of numerous inflammatory factors and other inflammatory components can cause abnormal glucose and lipid metabolism in local tissues, affecting local blood circulation and interfering wound repair $(9,10)$. At the same time, the internal and external toxins secreted by bacteria and the persistent damage caused by the bacterial capsule, if not removed in time, will form a vicious circle with a strong inflammatory response. The use of antibiotics to reasonably and effectively control infection can also be beneficial to the healing of local injury. Thus, reasonable control of UTI and local or even systemic inflammation before surgery is crucial to the protection of renal function among solitary kidney patients within 1 month after surgery. Diabetes and hypertension are common chronic diseases nowadays, which can lead to small vessel sclerosis and other lesions. Diabetes can cause peripheral nerve injury, reduce the release of neurotrophic factor, and sclerose local small vessel, leading to abnormal blood supply and interfering tissue repair $(11,12)$. Our study shows that combined diseases tend to deteriorate renal function after PCNL, even though the result is not significant. For the renal function at 6 months after PCNL, we found diabetes significantly deteriorated renal function, which may be related to impaired vascular function. Reasonable control of blood glucose level before and after surgery plays an important role in postoperative injury repair, so as to protect the renal function of patients. Interestingly, we found a reduction in post-operative renal function at 6 months in the operation time within $20 \mathrm{~min}$. It may be related to delayed hemorrhage to some degree. Admittedly, for these patients keeping their own lifestyle for long period, we cannot rule out that the intake of some substances unconducive to renal function or some combined diseases cause a deterioration of renal function. The group of operation duration beyond 40 min showed a tendency of renal function deterioration at 1 month after PCNL, and the main reasons can be long-time bleeding and exposure to infectious situation. The operation duration is neither as long nor as short as possible. Reasonable duration is very important for any operation. Strict control of operation time is conducive to shortening the duration of intraoperative bleeding and thus reducing the duration of continuous exposure to infectious factors and probable postoperative infection. With preoperative reasonable control of infection, PCNL after verification and confirmation of infection control may be relatively safe and reliable for solitary kidney patients, and postoperative infection management also need to be considered. It is still worth further discussion whether the patients with some combined diseases such as diabetes should receive surgery after effective and reasonable control of blood glucose level, otherwise they are not suitable for PCNL.

Due to the small number of solitary kidney patients receiving PCNL, and the decline in the number of staghorn calculus patients with the popularization of physical 


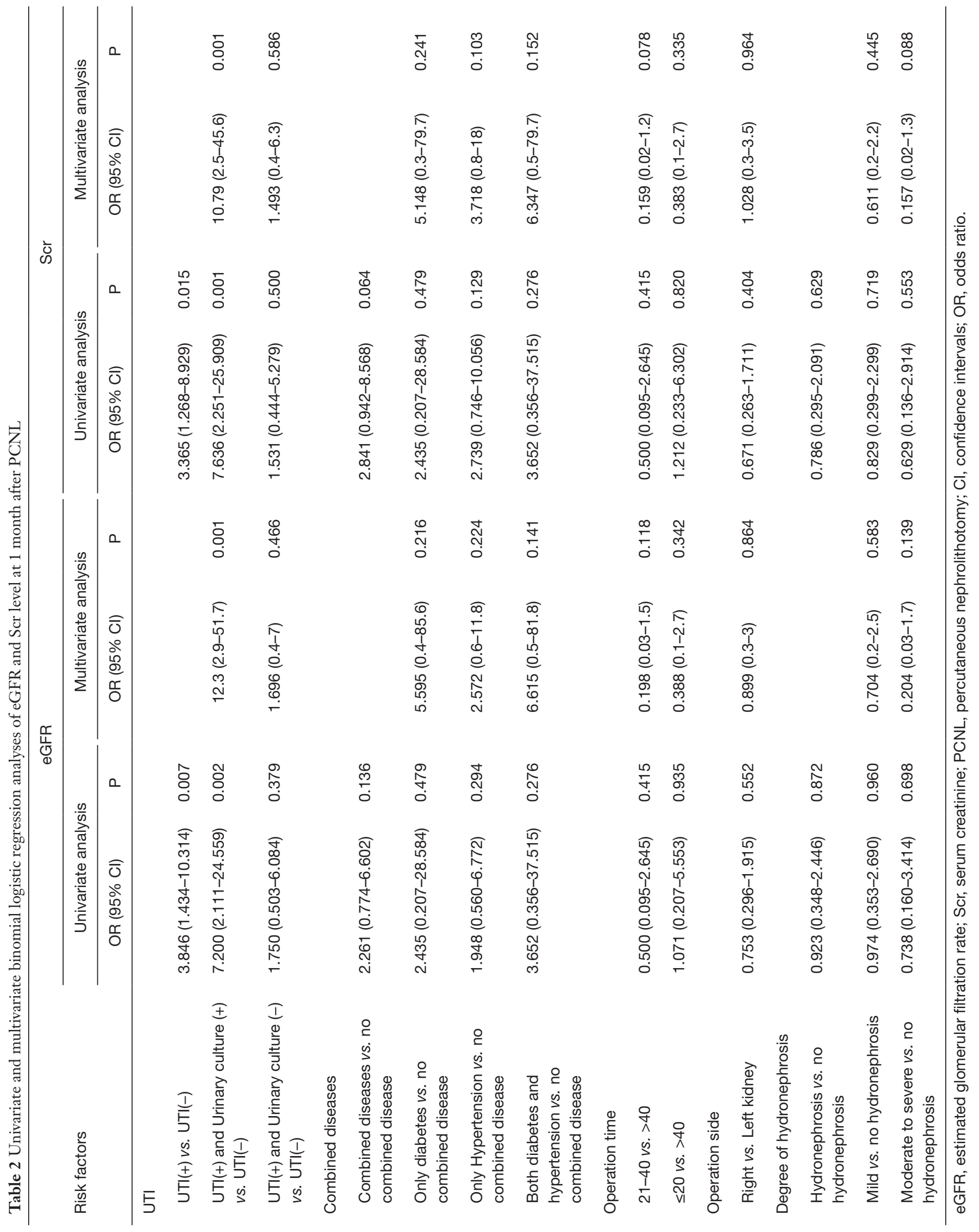




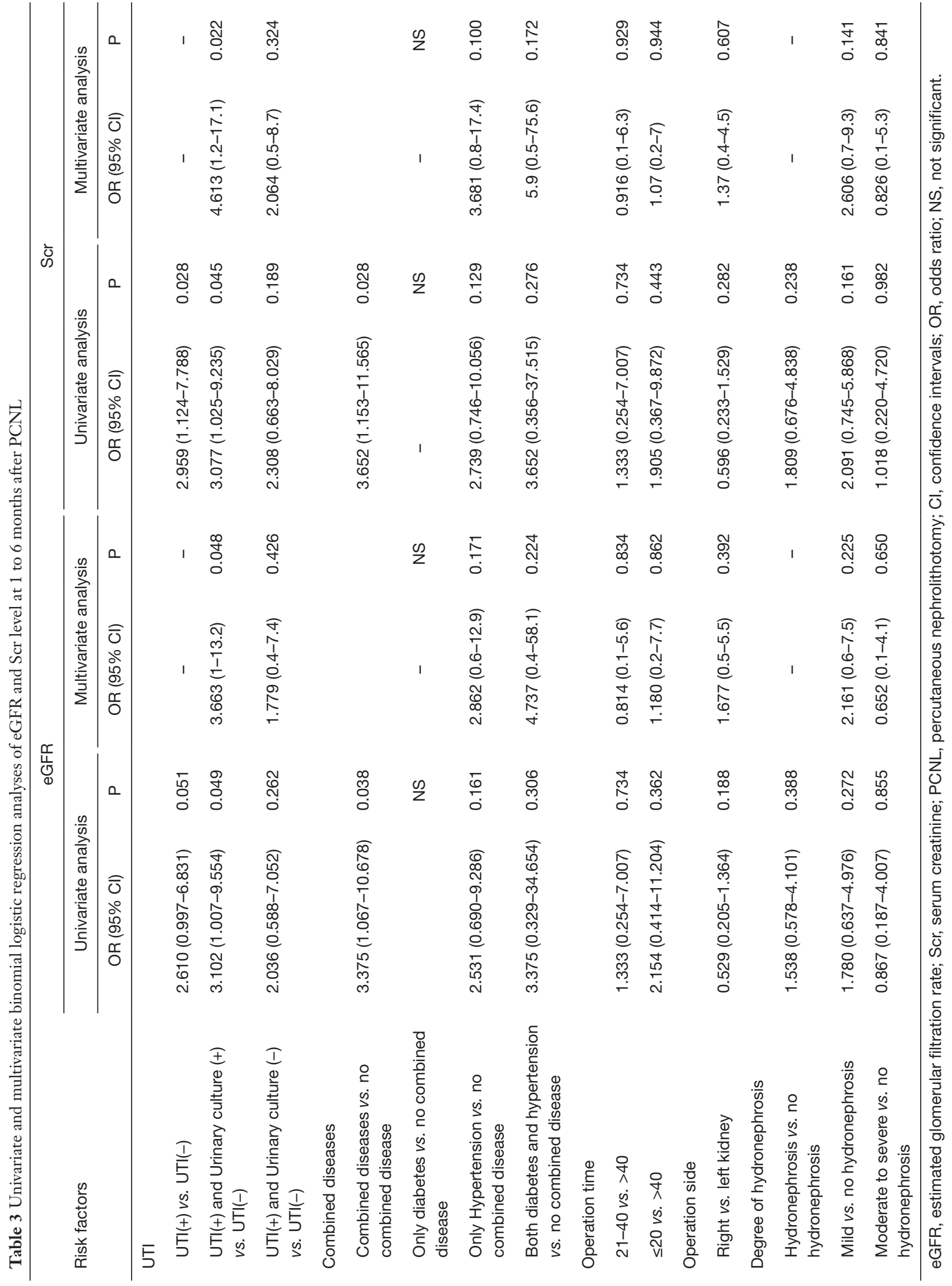


examination, there may be some potential deficiencies in this study.

\section{Conclusions}

UTI and combined diseases such as diabetes are the main factors deteriorating the postoperative renal function of patients. The operation duration, degree of hydronephrosis, and surgical side may also deteriorate renal function after surgery. Reasonable and effective preoperative preparation (e.g., infection control, treatment of combined diseases) and long-term postoperative follow-up play an important role in improving and protecting renal function after PCNL in solitary kidney patients with staghorn calculi.

\section{Acknowledgments}

Funding: This work was financed by grants from the 1.3.5 Project for Disiplines of Excellence, West China Hospital, Sichuan University (ZY2016104 and ZYGD18011) and Department of Science and Technology of Sichuan Province (2018SZ0168).

\section{Footnote}

Reporting Checklist: The authors have completed the STROBE reporting checklist. Available at http://dx.doi. org/10.21037/tau-20-916

Data Sharing Statement: Available at http://dx.doi. org/10.21037/tau-20-916

Conflicts of Interest: All authors have completed the ICMJE uniform disclosure form (available at http://dx.doi. org/10.21037/tau-20-916). The authors have no conflicts of interest to declare.

Ethical Statement: The authors are accountable for all aspects of the work in ensuring that questions related to the accuracy or integrity of any part of the work are appropriately investigated and resolved. The present study was conducted with the approval and supervised by the ethics committee of West China Hospital [registration number 2018(182)]. The study has conformed to the provisions of the Declaration of Helsinki (as revised in 2013). Because of the retrospective nature of the research, the requirement for informed consent was waived.
Open Access Statement: This is an Open Access article distributed in accordance with the Creative Commons Attribution-NonCommercial-NoDerivs 4.0 International License (CC BY-NC-ND 4.0), which permits the noncommercial replication and distribution of the article with the strict proviso that no changes or edits are made and the original work is properly cited (including links to both the formal publication through the relevant DOI and the license). See: https://creativecommons.org/licenses/by-nc-nd/4.0/.

\section{References}

1. Turk C, Petrik A, Sarica K, et al. EAU Guidelines on Diagnosis and Conservative Management of Urolithiasis. Eur Urol 2016;69:468-74.

2. van de Pol JAA, van den Brandt PA, Schouten LJ. Kidney stones and the risk of renal cell carcinoma and upper tract urothelial carcinoma: the Netherlands Cohort Study. Br J Cancer 2019;120:368-74.

3. Abdelhafez MF, Bedke J, Amend B, et al. Minimally invasive percutaneous nephrolitholapaxy (PCNL) as an effective and safe procedure for large renal stones. BJU Int 2012;110:E1022-6.

4. Besiroglu H, Merder E, Dedekarginoglu G. The safety and effectiveness of percutaneous nephrolithotomy in solitary kidney aging male patients: our single-center experience. Aging Male 2020;1-7.

5. Bruckner K. Renal Function: Guardian of Immune Homeostasis. Immunity 2019;51:596-8.

6. Massie AB, Holscher CM, Henderson ML, et al. Association of Early Postdonation Renal Function With Subsequent Risk of End-Stage Renal Disease in Living Kidney Donors. JAMA Surg 2020;155:e195472.

7. Bravi CA, Vertosick E, Benfante N, et al. Impact of Acute Kidney Injury and Its Duration on Long-term Renal Function After Partial Nephrectomy. Eur Urol 2019;76:398-403.

8. Bai YJ, Wang XM, Yang YB, et al. Percutaneous nephrolithotomy versus retrograde intrarenal surgery for the treatment of kidney stones up to $2 \mathrm{~cm}$ in patients with solitary kidney: a single centre experience. BMC Urol 2017;17:9.

9. Cooke JP. Inflammation and Its Role in Regeneration and Repair. Circ Res 2019;124:1166-8.

10. Simonetti O, Lucarini G, Morroni G, et al. New Evidence and Insights on Dalbavancin and Wound Healing in a Mouse Model of Skin Infection. Antimicrob Agents 
Chemother 2020;64:e02062-19.

11. Conway DS, Thompson NR, Cohen JA. Influence of hypertension, diabetes, hyperlipidemia, and obstructive lung disease on multiple sclerosis disease course. Mult

Cite this article as: Wang J, Bai Y, Yin S, Cui J, Tang Y, Wang Z, Chen B, Li H, Wei W, Wang J. Risk factors for deterioration of renal function after percutaneous nephrolithotomy in solitary kidney patients with staghorn calculi. Transl Androl Urol 2020;9(5):2022-2030. doi:10.21037/tau-20-916
Scler 2017;23:277-85.

12. Marrie RA, Kosowan L, Singer A. Management of diabetes and hypertension in people with multiple sclerosis. Mult Scler Relat Disord 2020;40:101987. 
Supplementary

Table S1 Baseline demographics of whole research (N=71)

\begin{tabular}{lc}
\hline Baseline demographics & Values \\
\hline Male:female & $53: 18$ \\
Age (years), mean (rang/SD) & $54.3(24-71 / 10.2)$ \\
Hypertension rate & $17(23.9 \%)$ \\
Diabetes rate & $7(9.9 \%)$ \\
CKD III-V & $19(26.8 \%)$ \\
Operative side (right:left kidney) & $35: 36$ \\
Degree of hydronephrosis (non:mild:moderate to severe) & $25: 37: 9$ \\
UTI (yes:no) & $38: 33$ \\
Urine culture (positive: negative) & $24: 47$ \\
Mean pre-op Scr ( $\mu$ mol/L), mean (rang/SD) & $130.9(61-243 / 41.0)$ \\
Mean pre-op eGFR (mL/min/1.73 m²), mean (rang/SD) & $55.1(20.3-97.5 / 18.9)$ \\
Diameter of largest stone (cm), mean (rang/SD) & $5.0(3-9.3 / 1.7)$ \\
Operative time (min), mean (rang/SD) & $28.7(10-60 / 13.3)$ \\
Stone free rate & 51 (71.8\%) \\
\hline
\end{tabular}

Pre-op, pre-operation; SD, standard deviation. 